\title{
Hair Bundle Defects and Loss of Function in the Vestibular End Organs of Mice Lacking the Receptor-Like Inositol Lipid Phosphatase PTPRQ
}

\author{
Richard J. Goodyear, ${ }^{1}$ Sherri M. Jones, ${ }^{2}$ Louise Sharifi, ${ }^{1}$ Andy Forge, ${ }^{3}$ and Guy P. Richardson ${ }^{1}$ \\ ${ }^{1}$ School of Life Sciences, University of Sussex, Falmer, Brighton BN1 9QG, United Kingdom, ${ }^{2}$ Department of Communication Sciences and Disorders, East \\ Carolina University, Greenville, North Carolina 27858, and ${ }^{3}$ University College London Ear Institute, University College London, London WC1 8EE, United \\ Kingdom
}

Recent studies have shown that mutations in $P T P R Q$, a gene encoding a receptor-like inositol lipid phosphatase, cause recessive, nonsyndromic, hereditary hearing loss with associated vestibular dysfunction. Although null mutations in Ptprq cause the loss of highfrequency auditory hair cells and deafness in mice, a loss of vestibular hair cells and overt behavioral defects characteristic of vestibular dysfunction have not been described. Hair bundle structure and vestibular function were therefore examined in Ptprq mutant mice. Between postnatal days 5 and 16, hair bundles in the extrastriolar regions of the utricle in Ptprq ${ }^{-1-}$ mice become significantly longer than those in heterozygous controls. This increase in length (up to 50\%) is accompanied by the loss and fusion of stereocilia. Loss and fusion of stereocilia also occurs in the striolar region of the utricle in Ptprq ${ }^{-1-}$ mice, but is not accompanied by hair bundle elongation. These abnormalities persist until 12 months of age but are not accompanied by significant hair cell loss. Hair bundle defects are also observed in the saccule and ampullae of $\mathrm{Ptprq}^{-1-}$ mice. At $\sim 3$ months of age, vestibular evoked potentials were absent from the majority (12 of 15) of Ptprq ${ }^{-1-}$ mice examined, and could only be detected at high stimulus levels in the other 3 mutants. Subtle but distinct defects in swimming behavior were detected in most (seven of eight) mutants tested. The results reveal a distinct phenotype in the vestibular system of Ptprq ${ }^{-1-}$ mice and suggest similar hair bundle defects may underlie the vestibular dysfunction reported in humans with mutations in PTPRQ.

\section{Introduction}

Deafness is one of the most common genetic diseases, and mutations in $>75$ genes have been identified thus far that affect the function of the inner ear (Hereditary Hearing Loss Homepage; http://hereditaryhearingloss.org). Many forms of human hereditary deafness are due to mutations that disrupt the structure and function of the hair bundle, a mechanosensory structure located at the apical pole of the hair cell. This structure is composed of two or more height-ranked rows of stereocilia and, in most organs of the inner ear, a single kinocilium (Schwander et al., 2010). A variety of links (tip links, top connectors, shaft connectors, and ankle links) interconnect the stereocilia, and the kinocilium is attached to the nearest stereocilia by kinocilial links (Nayak et al., 2007).

Mutations in the genes encoding most of the proteins associated with these links cause deafness. For example, cadherin-23 and

\footnotetext{
Received July 15, 2011; revised Jan. 4, 2012; accepted Jan. 5, 2012.

Author contributions: R.J.G., S.M.J., and G.P.R. designed research; R.J.G., S.M.J., L.S., A.F., and G.P.R. performed research; R.J.G. and S.M.J. analyzed data; R.J.G., S.M.J., and G.P.R. wrote the paper.

This work was supported by Wellcome Trust Grant 087737, Deafness Research United Kingdom, the Royal National Institute for Deaf People (now Action on Hearing Loss), and NIH Grant R01 DC006443. We thank Lindsey Welstead and Kevin Legan for genotyping the animals, and Liz Croskery for assistance with VsEP data analysis.

Correspondence should be addressed to Dr. Guy P. Richardson, School of Life Sciences, University of Sussex, Falmer, Brighton BN1 9QG, UK. E-mail: g.p.richardson@sussex.ac.uk.

DOI:10.1523/JNEUROSCI.3635-11.2012

Copyright $\odot 2012$ the authors $\quad 0270-6474 / 12 / 322762-11 \$ 15.00 / 0$
}

protocadherin-15 are products of the Usher deaf-blind syndrome genes USH1D (Bork et al., 2001) and USH1F (Ahmed et al., 2001; Alagramam et al., 2001), respectively, and together these two proteins form both the tip and the kinocilial links (Siemens et al., 2004; Söllner et al., 2004; Ahmed et al., 2006; Kazmierczak et al., 2007; Goodyear et al., 2010). Stereocilin, a product of the DFNB16 locus (Verpy et al., 2001), is associated with the top connectors of mammalian outer hair cells (Verpy et al., 2008, 2011), and the USH2C gene encoding the very large G-protein-coupled receptor Vlgr1 (Weston et al., 2004) is a component of the ankle links of the hair bundle (McGee et al., 2006; Michalski et al., 2007).

The receptor-like inositol lipid phosphatase Ptprq (Oganesian et al., 2003) is associated with the shaft connectors, and mice with effective null mutations in Ptprq have defects in their auditory hair bundles that become apparent within the first $2 \mathrm{~d}$ after birth (Goodyear et al., 2003). By P8, many stereocilia are missing from the hair bundles of both inner and outer hair cells in the Ptprq $^{-1-}$ mouse, and the hair bundles of the inner hair cells typically have a few elongated and fused stereocilia, while those of the outer hair cells have stereocilia that are shorter than those in heterozygous controls (Goodyear et al., 2003). The hair cells in the basal, high-frequency region of the Ptprq $^{-1-}$ mutant cochlea eventually disappear and a Preyer reflex can no longer be detected in response to a loud $20 \mathrm{kHz}$ tone burst.

Two recent studies (Schraders et al., 2010; Shahin et al., 2010) have revealed that mutations in PTPRQ cause autosomal, non- 

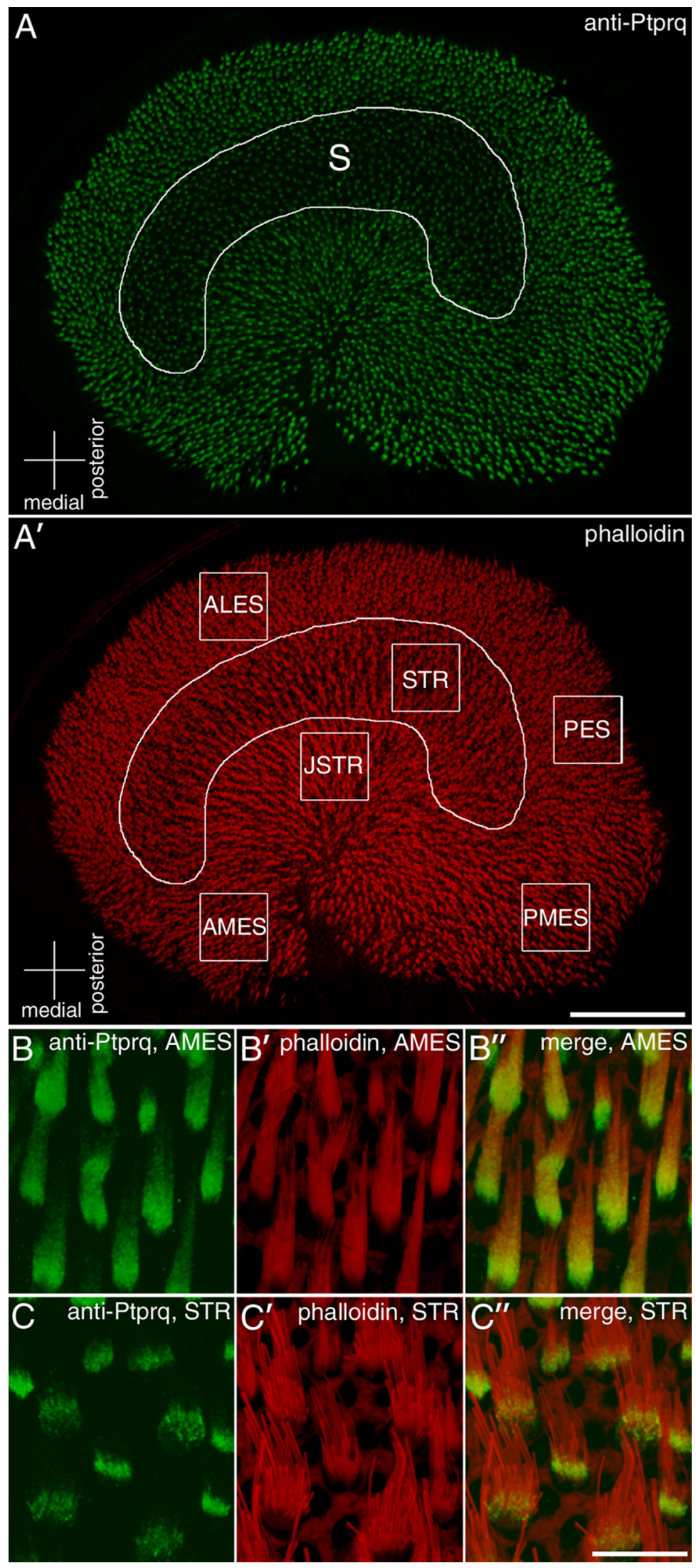

Figure 1. Utricular regions of interest and regional variations in the distribution of Ptprq. Anti-Ptprq $(\boldsymbol{A}-\boldsymbol{C})$ and phalloidin $\left(\boldsymbol{A}^{\prime}-\boldsymbol{C}^{\prime}\right)$ staining in the mature (P42) utricle from a Ptprq ${ }^{+/-}$mouse. The curved line in $\boldsymbol{A}$ and $\boldsymbol{A}^{\prime}$ delineates the striolar region (S), in which anti-Ptprq labeling is weak compared with the surrounding, extrastriolar region. The boxes in $\boldsymbol{A}^{\prime}$ represent regions used for subsequent analysis. Anterior lateral extrastriolar (ALES), striolar (STR), juxtastriolar (JSTR), posterior extrastriolar (PES), anterior medial extrastriolar (AMES), and posterior medial extrastriolar (PMES) regions were chosen. High-magnification images from syndromic recessive deafness at the DFNB84 locus in humans. Although Ptprq $^{-1-}$ mice do not exhibit any obvious, overt indications of vestibular dysfunction, the affected patients in two of the three DFNB84 families are reported to have a vestibular disorder, in addition to deafness. These findings prompted us to examine the structure and function of the vestibular end organs in Ptprq ${ }^{-1-}$ mice. A distinctive phenotype is revealed that may underlie the vestibular defect reported in DFNB84 patients.

\section{Materials and Methods}

Mice. Ptprq-CAT-ko mice, in which a neomycin resistance cassette replaces a 1061 bp genomic fragment encompassing a part of the intracellular domain of Ptprq that includes the catalytic site, were originally produced by and obtained from Drs. Dan Bowen-Pope and Ron Seifert (University of Seattle, Seattle, WA). The Ptprq-CAT-ko mice were on a mixed (50:50) C57BL/6J:129/Sv background and were the progeny of matings set up between heterozygous Ptprq-CAT-ko mice, or heterozygous and homozygous Ptprq-CAT-ko mice. Mice homozygous for this mutation are functional nulls and referred to as Ptprq ${ }^{-1-}$ mice. Mice with a deletion of the region encoding the seven transmembrane and cytoplasmic domains of the very large G-protein-coupled receptor, Vlgr1, were obtained from Dr. Perrin White (University of Texas Southwestern Medical Center, Dallas, TX) (McMillan and White, 2004). These Vlgr1/del7TM mice were on a mixed (50:50) C57BL/6J $\times 129 \mathrm{X} / \mathrm{Sv}$ background. Mice homozygous for this deletion, Vlgr1 $1^{\text {del7TM/del7TM }}$ mice, do not express any protein that can be detected with antibodies raised to the intracellular domain of Vlgr 1 and lack ankle links (McGee et al., 2006). Ptprq $^{-1}$ and $V l g r 1^{\text {del7TM/del } 7 T M}$ mice were crossed to produce double heterozygotes, and these were then inbred to produce Ptprq ${ }^{-1-} / V_{l g r} 1^{+/ d e l 7 T M}$ and $P t p r q^{+/-} /$Vlgr $1^{\text {del7TM/dellTM }}$ mice that were mated to produce the double mutants used in this study. Mice of either sex were used in this study, and all mice were genotyped using primers as described previously (McMillan and White, 2004; McGee et al., 2006). Mice were housed in the United Kingdom in accordance with Home Office guidelines. All procedures involving animals excepting those performed at East Carolina University were performed with the authority of the United Kingdom Home Office and with the approval of the local ethical committee of the University of Sussex.

$\leftarrow$

AMES $(\boldsymbol{B})$ and STR ( $\boldsymbol{C}$ regions show the relative distributions of Ptprq on extrastriolar $(\boldsymbol{B})$ and striolar $(\boldsymbol{C})$ hair bundles. AntiPtprq staining extends along most of the length of bundles of extrastriolar hair cells $(\boldsymbol{B})$ but is restricted to the base of bundles in the striolar region (C). Scale bars: $\boldsymbol{A}^{\prime}, 100 \mu \mathrm{m}$; (in $\boldsymbol{C}^{\prime \prime}$ ) $B-C^{\prime \prime}, 10 \mu \mathrm{m}$. 
Confocal microscopy. For confocal microscopy, inner ears were placed in PBS ( $150 \mathrm{~mm} \mathrm{NaCl}, 10 \mathrm{~mm}$ sodium phosphate, $\mathrm{pH}$ 7.4) and, after removal of the round and oval windows, immersion fixed in $3.7 \%$ formaldehyde ( $10 \%$ commercial formalin) in $0.1 \mathrm{~m}$ sodium phosphate, $\mathrm{pH}$ 7.2, initially for $2-4 \mathrm{~h}$ at room temperature and then overnight at $4^{\circ} \mathrm{C}$. Following three washes in PBS, the utricle (with attached lateral and anterior ampullae) was carefully dissected from the bony labyrinth, the epithelial roof overlying the macula was removed to expose the otoconia, and the samples were transferred in the enlarged tip of a serumprewetted, plastic micropipette into a small plastic Petri dish containing $3 \mathrm{ml}$ of $0.5 \mathrm{~m}$ EDTA, pH 8.0. After $30 \mathrm{~min}$ at room temperature, a time that was just sufficient for the majority of the otoconia to become translucent, samples were transferred into a $1.5 \mathrm{ml}$ microfuge tube containing $0.5 \mathrm{ml}$ of preblock [ $10 \%$ heat-inactivated horse serum, $0.1 \%$ Triton X-100 (TX-100) in PBS]. After one wash with preblock, Texas Redconjugated phalloidin was added to a final dilution of 1:500 to label F-actin, and the samples were incubated, with agitation, overnight. For labeling Ptprq in samples from heterozygous animals, tissues were double labeled with phalloidin and rabbit anti-Ptprq (R27; an antibody raised to the recombinant intracellular domain of avian Ptprq) (Nayak et al., 2011). Anti-Ptprq was subsequently detected with Alexa 488conjugated goat anti-rabbit Ig (Invitrogen; 1:500 dilution). Following labeling, tissues were washed three times in PBS containing $0.1 \%$ TX100 , three times in PBS, and mounted under a no. 0 thickness, round glass coverslip on a microscope slide using a small $(\sim 25 \mu \mathrm{l})$ volume of Vectashield mounting medium. Shims were not used and the tissues were allowed to flatten as the forces of surface tension pulled the coverslip down onto the slide. Nail varnish was used to finally seal the coverslip onto the slide, and the samples were imaged using a $100 \times$ oil-immersion lens, NA 1.4, using a Zeiss LSM 510 META confocal microscope.

Quantification of bundle lengths, areas, and numbers were obtained from $1024 \times 1024$ pixel compressed confocal $z$-series of flattened phalloidin-stained utricles using a $1.4 \mathrm{NA}, 100 \times$ oil-immersion lens and a $1.5 \times$ zoom factor. Values were measured for (typically) 10 hair bundles per image, with images from utricles of three different animals for each age, region, and genotype. Analysis of images was performed using the quantification tools available in Photoshop CS4. Hair bundle lengths were measured from the middle of the base of the bundle to the tip of the tallest stereocilia. Differences with previously published values for hair bundle height in the mouse utricle (Li et al., 2008) may be due to genetic background or the methods used to evaluate hair bundle height. Clearly immature or very short hair bundles were not chosen for measurement. Bundle areas were calculated by carefully drawing around the profile of each hair bundle with the polygonal lasso tool in Photoshop using, where possible, the same hair cells as those from which bundle lengths were measured. Hair bundle numbers were counted from the full field of $1024 \times 1024$ pixels at 1.5 zoom with the $100 \times$ lens $\left(=3715 \mu \mathrm{m}^{2}\right)$, counting only those hair cells where the entire bundle base was visible. Statistical significance was assessed using one-way ANOVA followed by the Tukey test.

Electron microscopy. For electron microscopy, inner ears were prepared as described above and immersion fixed in $2.5 \%$ glutaraldehyde in $0.1 \mathrm{M}$ sodium cacodylate buffer, $\mathrm{pH}$ 7.2. For transmission electron microscopy (TEM), 1\% tannic acid was added to the glutaraldehyde fixative. Samples were fixed as described above, initially at room temperature and then overnight at $4^{\circ} \mathrm{C}$. For scanning electron microscopy, the otoconial membrane was removed after the primary fixation by treating the samples with $50 \mu \mathrm{g} / \mathrm{ml}$ subtilisin (Protease XXIV; Sigma-Aldrich) in PBS for $30 \mathrm{~min}$ at room temperature. Samples were then postfixed in $1 \%$ osmium tetroxide in $0.1 \mathrm{~m}$ sodium cacodylate, $\mathrm{pH} 7.4$, for $\sim 2 \mathrm{~h}$ at room temperature, washed three times with $0.1 \mathrm{~m}$ sodium cacodylate buffer, pH 7.2, once with $\mathrm{H}_{2} \mathrm{O}$, and dehydrated through a series of ascending concentrations of ethanol. For scanning electron microscopy (SEM), samples were critical point dried from liquid $\mathrm{CO}_{2}$, mounted on aluminum stubs with double-sided sticky pads, sputter coated with gold, and viewed with a Leica Leo scanning electron microscope operating at $20 \mathrm{kV}$. For TEM, samples were equilibrated in propylene oxide, infiltrated with, and finally imbedded in, TAAB epoxy resin (Epon). Thin ( $\sim 90-\mathrm{nm}-$ thick) sections were cut with a diamond knife, mounted on copper mesh
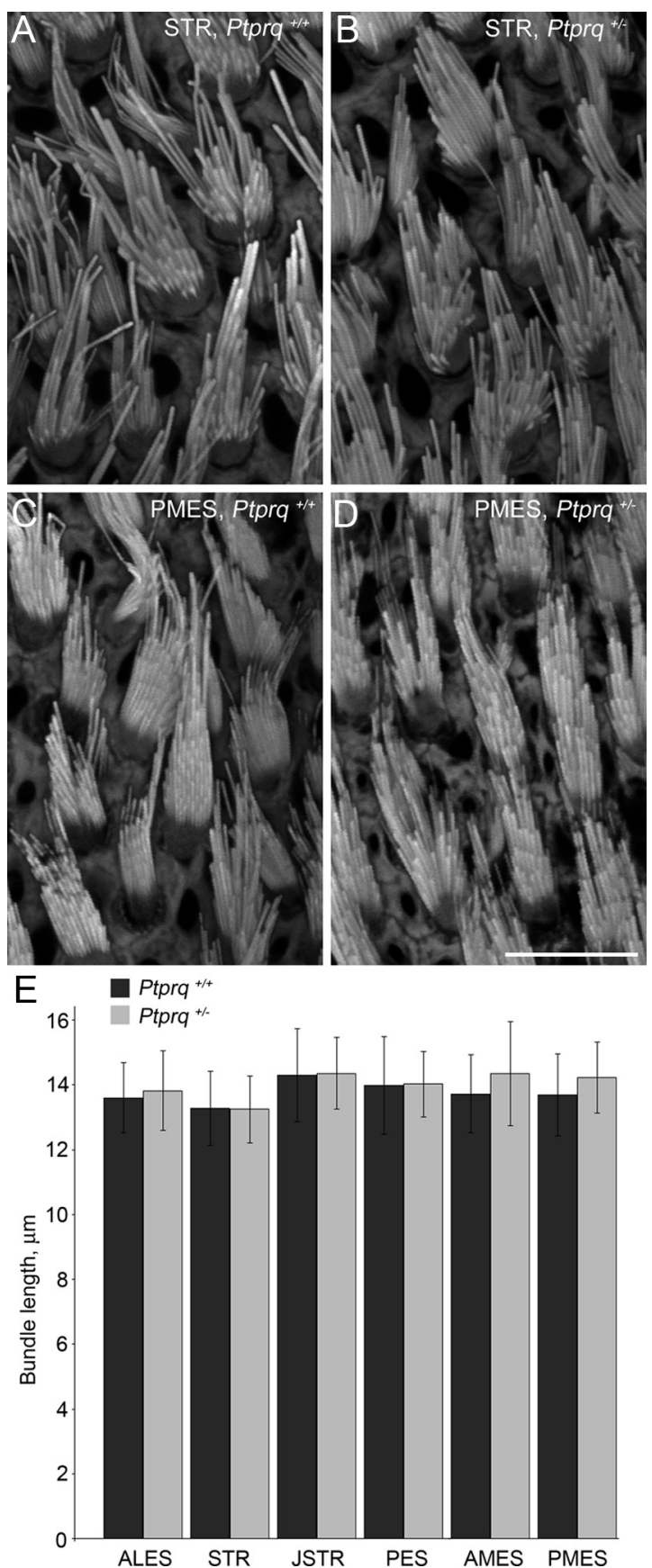

Figure 2. Appearance and length quantification of hair bundles from wild-type mice and Ptprq $^{+1}$ - littermates. Selected regions from phalloidin-stained whole mounts of wild-type $(\boldsymbol{A}, \boldsymbol{C})$ and Ptprq $^{+1-}(\boldsymbol{B}, \boldsymbol{D})$ utricles at P42. Hair bundles had an identical appearance in wildtype and Ptprq ${ }^{+1-}$ animals. Quantification of hair bundle length from all six regions used in this study demonstrated no significant differences in hair bundle height between wild-type and Ptprq $^{+I-}$ mice $(\boldsymbol{E})$. Scale bar: (in $\left.\boldsymbol{D}\right) \boldsymbol{A}-\boldsymbol{D}, 10 \mu \mathrm{m}$.

grids, double stained with uranyl acetate and lead citrate (Reynolds, 1963), and viewed in a Hitachi 7100 electron microscope at an operating voltage of $100 \mathrm{kV}$. Images were captured with a Gatan digital camera.

Measurement of vestibular evoked potentials. The use of animals for vestibular functional measures was approved by the Institutional Animal Care and Use Committee at East Carolina University. Vestibular evoked potential (VsEP) recordings were based on methods for mice (Jones et al., 1999, 2004; Mock et al., 2011) and are briefly described here. Animals (2-4 months of age; Ptprq $^{-1-}, n=15 ;$ Ptprq $^{+/-}, n=15 ;$ Ptprq $^{+/+}, n=$ 4) were anesthetized with ketamine/xylazine $(18: 2 \mathrm{mg} / \mathrm{ml}), 7 \mu \mathrm{l}$ per gram body weight, injected intraperitoneally. Linear acceleration pulses, $2 \mathrm{~ms}$ 

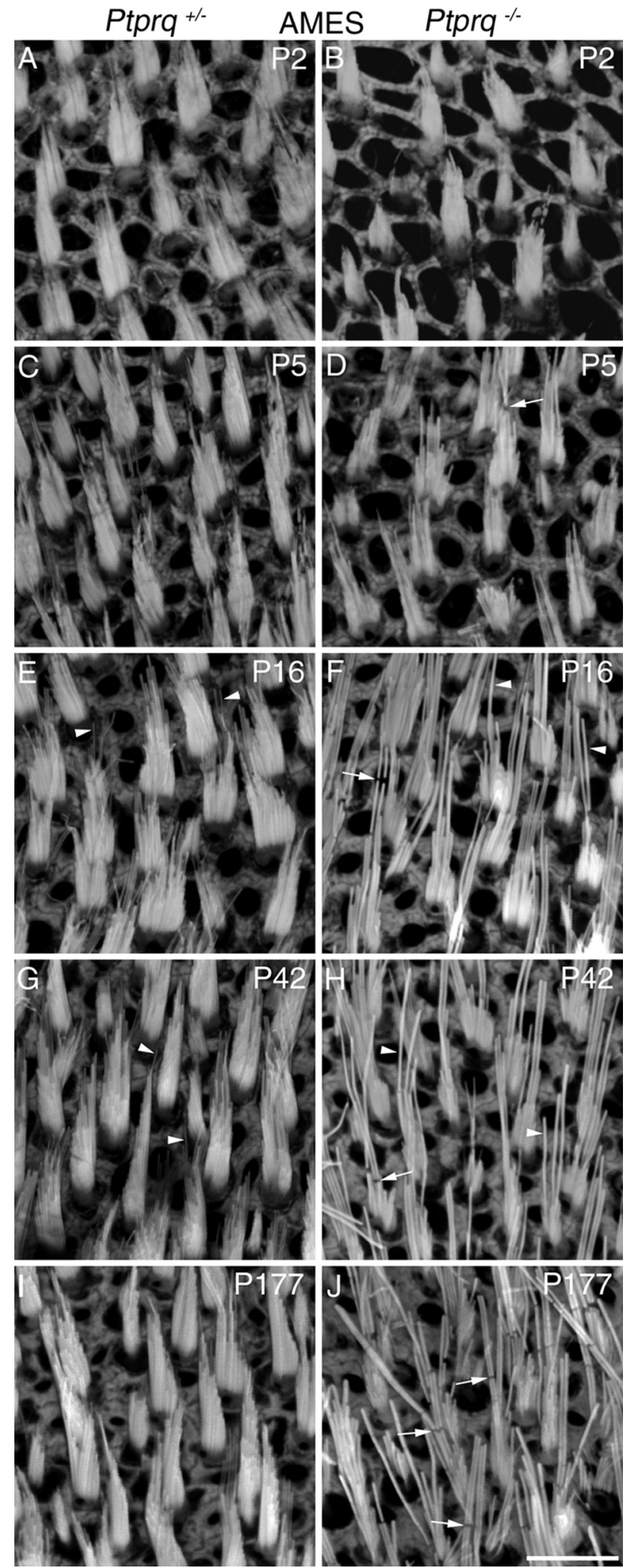

Figure 3. Hair bundle morphology in the anterior medial extrastriolar region of $\mathrm{Ptprq}^{+/-}$and Pt$\mathrm{prq}^{-1-}$ mice. Phalloidin-stained hairbundlesfrom the AMES region of themouseutricle, atP2 $(A, B), P 5(C$,

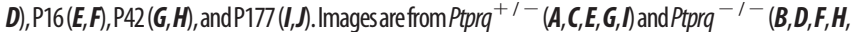
1) animals. Clearly evident from $P 16$, Ptprq $^{-1-}$ hair bundles have fewer, thicker stereocilia that appear taller, compared with those of $P$ tprq ${ }^{+/-}$mice. The arrowheads in $\boldsymbol{E}, \boldsymbol{F}$, and $\mathbf{G}, \boldsymbol{H}$, highlight the difference in thickness between stereocilia of $P$ tprq ${ }^{+/-}(\boldsymbol{E}, \boldsymbol{G})$ and $P_{t p r q}{ }^{-1-}(\boldsymbol{F}, \boldsymbol{H})$ mice. The arrows in $\boldsymbol{D}, \boldsymbol{F}, \boldsymbol{H}$, and $\boldsymbol{J}$ indicate breaks in the F-actin staining, particularly evident in $P$ tprq ${ }^{-1-}$ hair bundles. Scale bar: (in $\left.\mathcal{A}\right)$ A-J, $10 \mu \mathrm{m}$
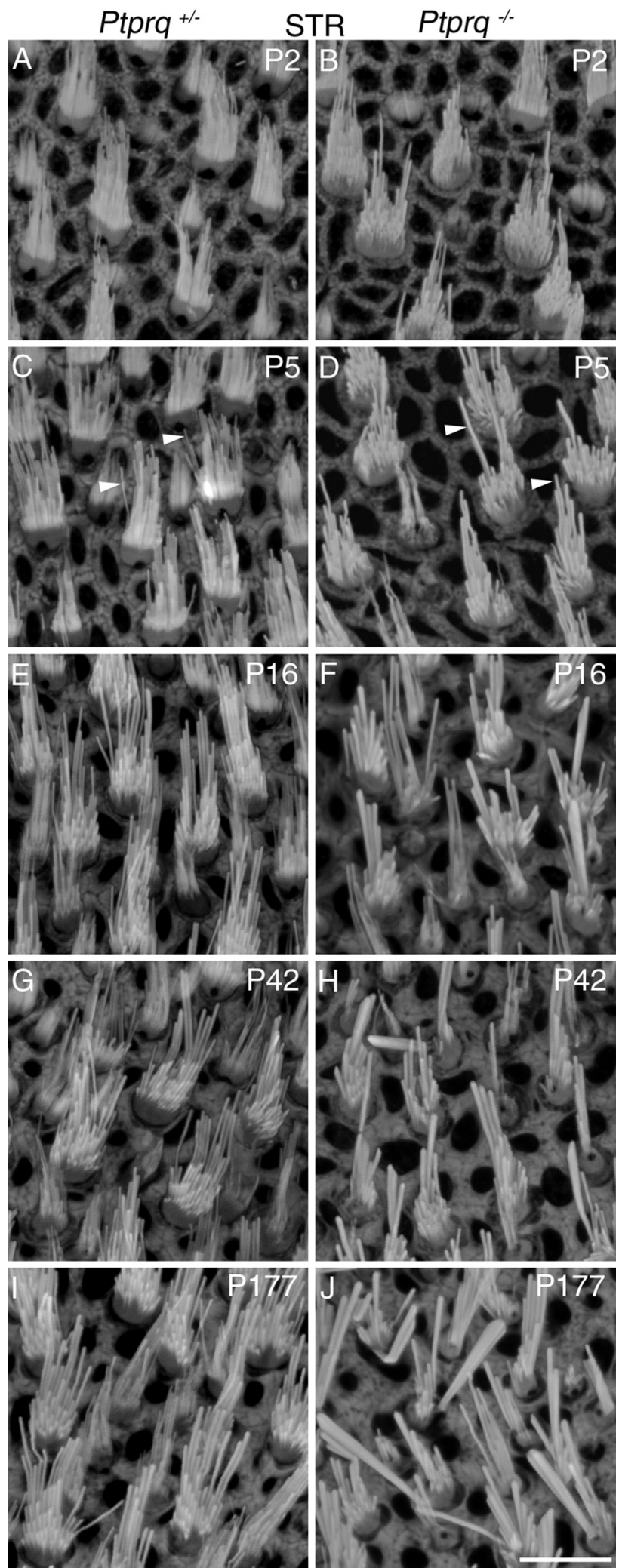

Figure 4. Hair bundle morphology in the striolar region of $\mathrm{Ptprq}^{+/-}$and $\mathrm{Ptprq}^{-1-}$ mice Phalloidin-stained hair bundles from the STR region of the mouse utricle at $\mathrm{P2}(A, B), P 5(C, D)$, P16 $(\boldsymbol{E}, \boldsymbol{F}), \mathrm{P} 42(\boldsymbol{G}, \boldsymbol{H})$, and P177 $(\boldsymbol{I}, \boldsymbol{J})$. Images are from Ptprq ${ }^{+/-}(\boldsymbol{A}, \boldsymbol{C}, \boldsymbol{E}, \boldsymbol{G}, \boldsymbol{I})$ and Ptprq $(\boldsymbol{B}, \boldsymbol{D}, \boldsymbol{F}, \boldsymbol{H}, \boldsymbol{J})$ animals. Hair bundles of Ptprq ${ }^{-1-}$ animals have noticeably fewer, thicker stereocilia than those of $\mathrm{Ptprq}^{+/-}$animals in this region of the utricle. The arrowheads in $C$ and $D$ indicate examples of individual stereocilia and highlight the difference in thickness. Scale bar: (in J) $A-J, 10 \mu \mathrm{m}$. 

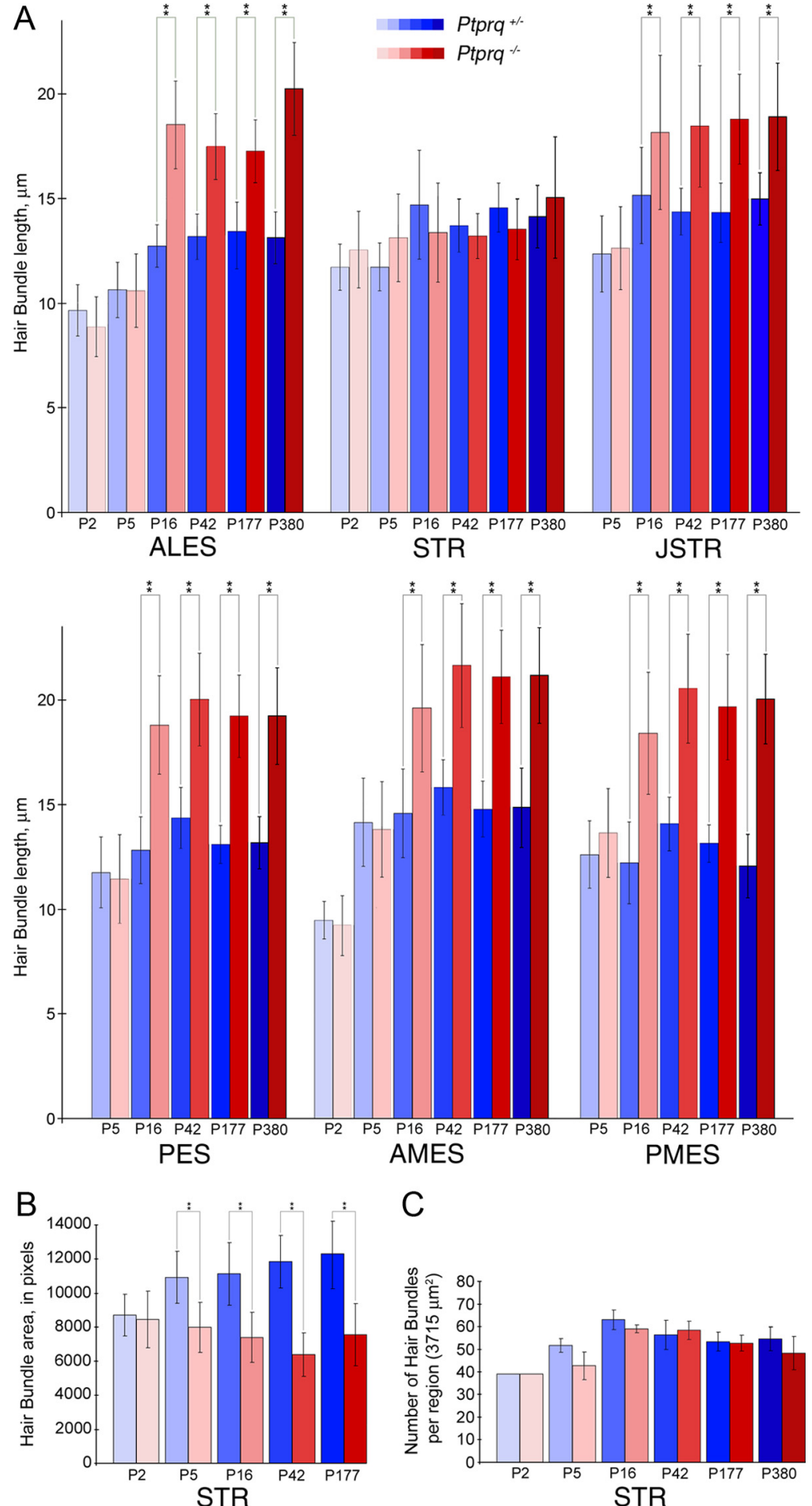

C
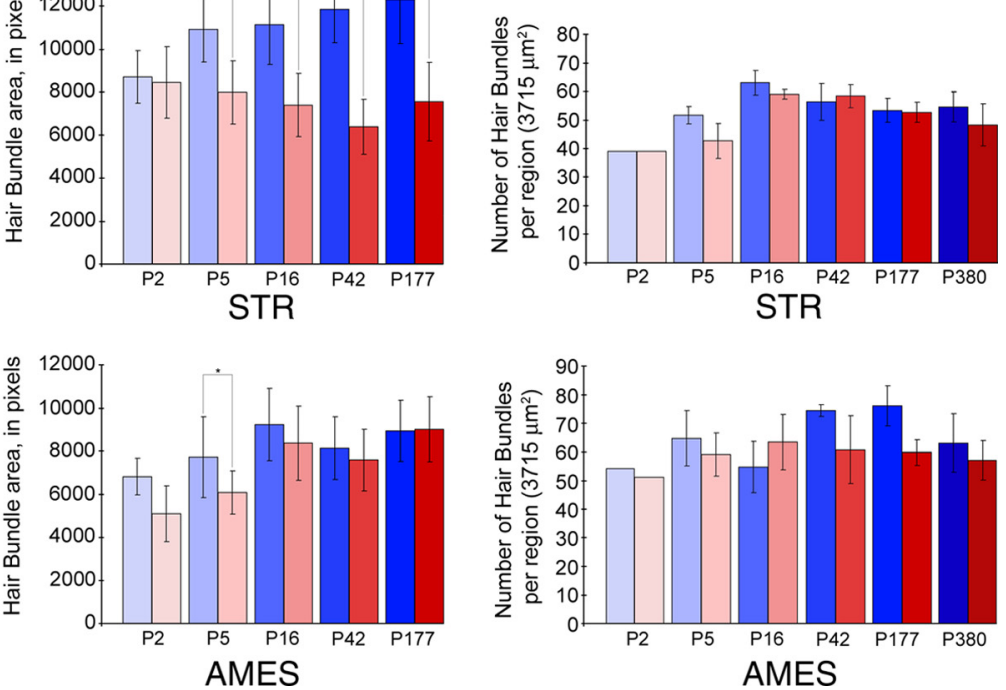

Figure 5. Quantification of hair bundle features and numbers in Ptprq ${ }^{+}-$and $^{\text {Ptprq }}{ }^{-1-}$ mice. Comparisons of hair bundle lengths $(\boldsymbol{A})$, profile areas $(\boldsymbol{B})$, and numbers $(\boldsymbol{C})$ at P2, P5, P16, P42, P177, and P380 in Ptprq ${ }^{+1-}$ and Ptprq ${ }^{-1-}$ mice. Data are from selected regions of interest. Hair bundles become significantly longer $(\boldsymbol{A})$ from P16 onward in all extrastriolar regions (ALES, duration, were presented to the cranium via a noninvasive spring clip that encircled the head anterior to the pinna and secured the head to a voltage-controlled mechanical shaker. Stimuli were presented along the naso-occipital axis using two stimulus polarities, normal $(+\mathrm{Gx}$ axis) and inverted ( $-\mathrm{Gx}$ axis). Stimuli were presented at a rate of 17 pulses/s and ranged from a maximum of $+6 \mathrm{~dB}$ to a minimum of $-18 \mathrm{~dB}$ re: $1.0 \mathrm{~g} / \mathrm{ms}$ (where $1 \mathrm{~g}=9.8 \mathrm{~m} / \mathrm{s}^{2}$ ) adjusted in $3 \mathrm{~dB}$ steps. Traditional signal averaging was used to resolve responses in electrophysiological recordings. Stainless-steel wire was placed subcutaneously at the nuchal crest to serve as the noninverting electrode. Needle electrodes were placed posterior to the right pinna and at the left hip for inverting and ground electrodes, respectively. Ongoing electroencephalographic activity was amplified $(200,000 \times)$, filtered $(300-3000 \mathrm{~Hz})$, and digitized (100 kHz sampling rate). A total of 256 primary responses was averaged for each VsEP response waveform. All responses were replicated. A VsEP intensity series was collected beginning at the maximum stimulus intensity (i.e., $+6 \mathrm{~dB}$ re: $1.0 \mathrm{~g} / \mathrm{ms}$ ) with and without acoustic masking $(50-50,000 \mathrm{~Hz}$ forward masker at $94 \mathrm{~dB}$ SPL), and then descending in 3 $\mathrm{dB}$ steps until no response was visible. Thresholds (measured in decibels re: $1.0 \mathrm{~g} / \mathrm{ms}$ ), peak latencies (in microseconds), and peak to peak amplitudes (in microvolts) were obtained from the VsEP waveform. Response parameters for the first response peak (P1 known to be generated by the peripheral eighth nerve) were compared between heterozygotes and homozygotes using independent sample $t$ tests.

Behavioral assessment of vestibular function. Mice underwent reaching reflex, Rotarod, and swimming tests to assess vestibular function. To test the reaching reflex, each mouse was held by its tail and lowered toward a surface. Mice with impaired vestibular function tend to curl toward their tail, but normal mice tend to stretch their paws out toward the approaching surface. The mice were subsequently tested on a Rotarod apparatus over a period of $4 \mathrm{~d}$. On the first day, the mice underwent training to familiarize themselves with the equipment. They were trained at a speed of $4 \mathrm{rpm}$, and the speed was then increased in $2 \mathrm{rpm}$ intervals every $60 \mathrm{~s}$ up to a speed of 10

JSTR, PES, AMES, and PMES) in Ptprq ${ }^{-1-}$ mice relative to heterozygous controls. In the STR region, average hair bundle length in Ptprq ${ }^{-1-}$ mice does not deviate greatly from that of Ptprq $^{+1-}$ mice at any stage examined. Significantly smaller average hair bundle areas $(\boldsymbol{B})$ are observed at early stages in the STR (P5) and AMES (P5) regions of Ptprq ${ }^{-1-}$ utricles, relative to heterozygous controls. At later stages (P16 onward), these differences become less apparent in the extrastriolar region but become more extreme in the striola. Significant differences in numbers of hair bundles present $(\boldsymbol{C})$ were not observed in any region examined. *Indicates that the value for $P t p r q^{-1-}$ is significantly different from the corresponding Ptprq ${ }^{+} /-$value at the $1 \%$ level. ${ }^{* *}$ Indicates significance at the $0.1 \%$ level. $p>0.05$ was considered nonsignificant. 

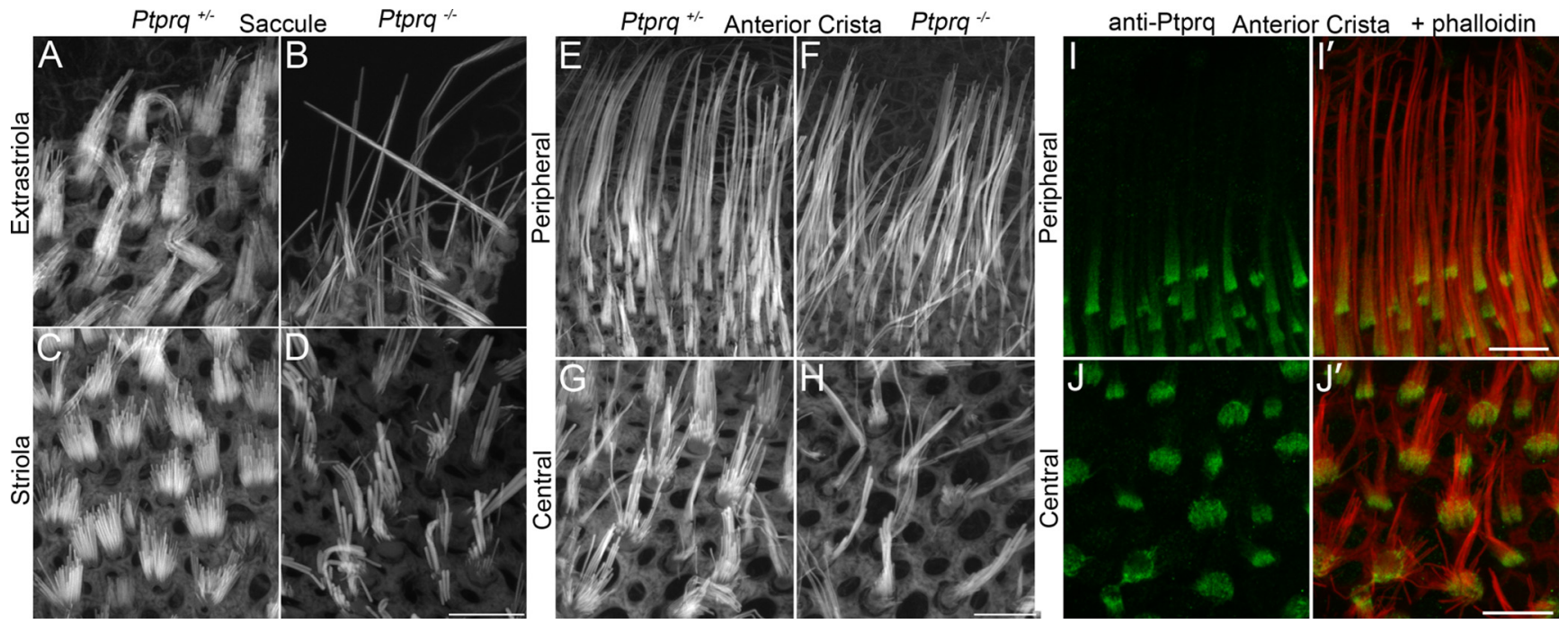

Figure 6. Hair bundle features in the saccular macula and crista ampullaris. Phalloidin-stained hair bundles from the extrastriolar $(\boldsymbol{A}, \boldsymbol{B})$ and striolar $(\boldsymbol{C}, \boldsymbol{D})$ regions of the saccule, and from the peripheral $(\boldsymbol{E}, \boldsymbol{F})$ and central $(\boldsymbol{G}, \boldsymbol{H})$ regions of the anterior crista of $\operatorname{Ptprq}^{+/-}(\boldsymbol{A}, \boldsymbol{C}, \boldsymbol{E}, \boldsymbol{G})$ and $\operatorname{Ptprq}^{-1-}(\boldsymbol{B}, \boldsymbol{D}, \boldsymbol{F}, \boldsymbol{H})$ animals at P177. Anti-Ptprq staining in the peripheral $\left(\boldsymbol{I}, \boldsymbol{I}^{\prime}\right)$ and central $\left(\boldsymbol{J}, \boldsymbol{J}^{\prime}\right)$ regions of the anterior cristae at P44 with phalloidin overlay shown in $I^{\prime}$ and $J^{\prime}$. Anti-Ptprq staining extends beyond the base of peripheral hair bundles but does not reach their tips. Anti-Ptprq staining is restricted to the base of central hair bundles. Scale bars: (in $\left.\mathbf{D}, \boldsymbol{H}, \boldsymbol{I}^{\prime}, \boldsymbol{J}^{\prime}\right) \mathbf{A}-\mathbf{D} ; \boldsymbol{E}-\boldsymbol{H} ; \boldsymbol{I}, \boldsymbol{I}^{\prime} ; \boldsymbol{J}, \boldsymbol{J}^{\prime}$, respectively, $10 \mu \mathrm{m}$.

rpm. Mice were replaced on the rod following each fall. On days 2, 3, and 4, the mice were tested at a constant speed of $10 \mathrm{rpm}$ for $180 \mathrm{~s}$. On days 3 and 4 , the mice were tested for a further $60 \mathrm{~s}$ at $15 \mathrm{rpm}$. The number of falls was recorded and the mice were replaced back on the rod immediately after each fall. To test swimming ability, the mice were placed in a container filled with water at $25^{\circ} \mathrm{C}$ for $1 \mathrm{~min}$. The mice were filmed during this period for subsequent analysis. Swimming behavior was classed as normal if the yellow belly was not visible during swimming and their backs were not frequently fully submerged. Rolling and excessive circling was noted and considered suggestive of vestibular dysfunction.

\section{Results}

Figure 1 provides a low-magnification survey image of the macula from a mature (6-week-old) heterozygous $\mathrm{Ptprq}^{+1-}$ mouse. Double labeling with antibodies to Ptprq (Fig. $1 A$, green) and phalloidin (Fig. $1 A^{\prime}$, red) reveals that hair bundles in the striolar region of the macula (Fig. $1 A, A^{\prime}$, delineated by the curved white line) stain weakly for Ptprq relative to those in the flanking extrastriolar regions. Regions of interest that were used in this study for quantitative analysis of hair bundle height, profile, and numbers at the different stages examined are indicated in Figure $1 A^{\prime}$ by white boxes. Examples of double-labeled hair bundles from two of these regions, the anterior medial extrastriolar region (AMES) and the striolar region (STR), are shown at higher magnification in Figure 1, $B$ and $C$. In the STR region, which contains broad hair bundles with relatively thick stereocilia, labeling for Ptprq is very much restricted to the basal region of the hair bundle (Fig. $1 C-C^{\prime \prime}$ ). In all of the extrastriolar regions, as exemplified by those in the AMES region, labeling for Ptprq is distributed over a larger extent of the surface of the hair bundle and extends further up toward the distal tip (Fig. $1 B-B^{\prime \prime}$ ). A difference between striolar and extrastriolar hair cells with regard to the distribution of Ptprq on the hair bundle has been previously described in the maculae of the chick inner ear (Goodyear and Richardson, 1992).

Mutations at the DNFB84 in humans are recessive, and no evidence has been found to suggest there is a heterozygous phenotype in the cochlea of Ptprq-CAT-ko mice. Nonetheless, we examined and compared hair bundle morphology and height in the striolar and extrastriolar regions of $\mathrm{Ptprq}^{+/+}$and $\mathrm{Ptprq}^{+1-}$
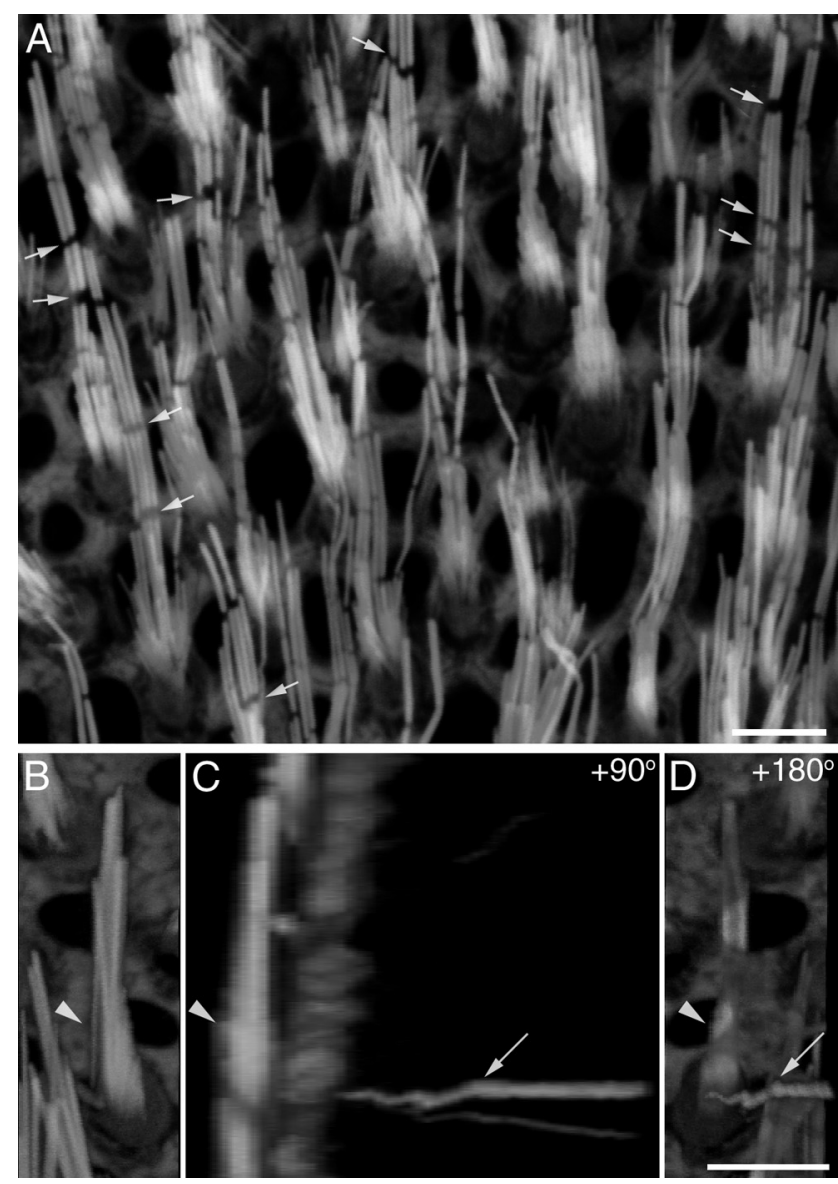

Figure 7. F-actin breaks and cytocauds in Ptprq ${ }^{-/}$- mice. Phalloidin-stained hair bundles from the juxtastriolar region of a P31 Ptprq ${ }^{-1-}$ utricle $(\boldsymbol{A})$ showing extensive breaks in F-actin staining of stereocilia. The breaks are often aligned with respect to each other in an individual hair bundle (arrows). Confocal images of a phalloidin-stained extrastriolar hair bundle from a P42 Ptprq $^{-1-}$ utricle (B-D). Compressed $z$-stacks showing a top surface view $(\boldsymbol{B})$, a $90^{\circ}$ rotation to give a side view $(\boldsymbol{C})$, and a $180^{\circ}$ rotation to give an underside view $(\boldsymbol{D})$ reveal that a cytocaud ( $\boldsymbol{C}, \boldsymbol{D}$, arrows) projects down from the base of the hair bundle $(\boldsymbol{B}-\boldsymbol{D}$, arrowhead) into the cell body. Scale bars: $\boldsymbol{A}, 5 \mu \mathrm{m}$; (in $\boldsymbol{D}) \boldsymbol{B}-\boldsymbol{D}, 5 \mu \mathrm{m}$. 

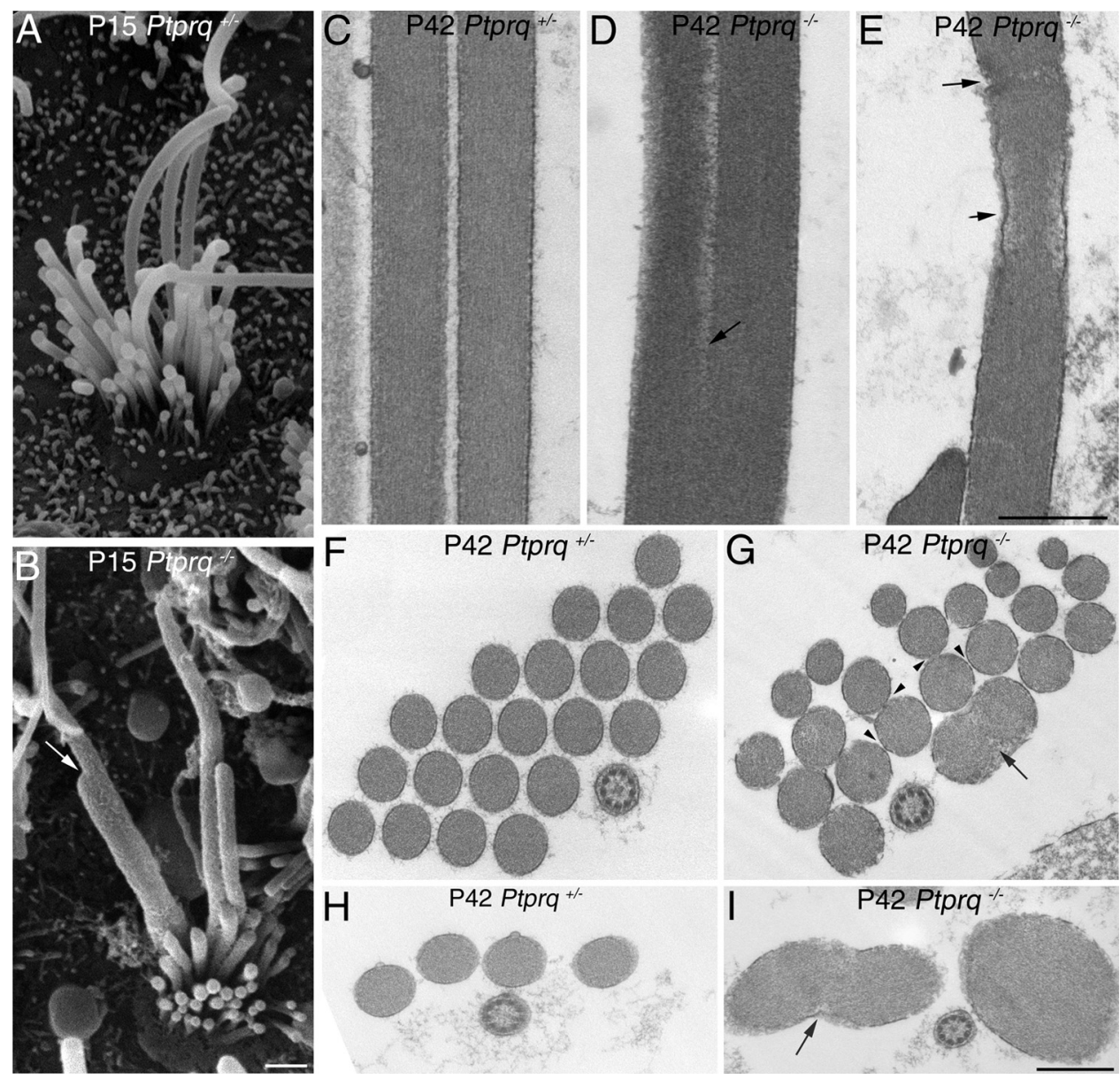

Figure 8. Scanning and transmission electron microscopy of $\mathrm{Ptprq}^{+/-}$and $P$ tprq ${ }^{-/-}$hair bundles. SEM images of extrastriolar hair bundles of the mouse utricle at P15, from Ptprq ${ }^{+/-}(\boldsymbol{A})$ and Ptprq $^{-1-}(\boldsymbol{B})$ mice. Evidence of fusion is observed between stereocilia in $P$ tprq ${ }^{-1-}$ mice (B, arrow). $\boldsymbol{C}-\boldsymbol{I}$, TEM images comparing stereocilia spacing and actin organization in hair bundles of Ptprq $^{+/-}(\boldsymbol{C}, \boldsymbol{F}, \boldsymbol{H})$ and $\operatorname{Ptprq}^{-I-}(\boldsymbol{D}, \boldsymbol{E}, \boldsymbol{G}, \boldsymbol{I})$ extrastriolar hair bundles of the $\mathrm{P} 42$ mouse utricle. Cross-sections through the lower $(\boldsymbol{F}, \boldsymbol{G})$ and upper $(\boldsymbol{H}, \boldsymbol{I})$ regions of hair bundles are shown in $\boldsymbol{F}-\boldsymbol{I}$. Stereocilia are more closely packed in $P$ tprq ${ }^{-I-}$ mice $(\boldsymbol{G}$, arrowheads) compared with heterozygous counterparts $(\boldsymbol{F})$ and display fusion $(\boldsymbol{D}, \boldsymbol{G}, \boldsymbol{I}$, arrows). 0 ccasional disruptions are seen in the actin filament core of Ptprq ${ }^{-1-}$ stereocilia (E, arrows). Scale bars: (in $\left.\boldsymbol{B}\right) \boldsymbol{A}, \boldsymbol{B}, 500 \mathrm{~nm}$; (in $\left.\boldsymbol{E}\right) \boldsymbol{C}-\boldsymbol{E}, 500 \mathrm{~nm}$; (in $\left.\boldsymbol{I}\right) \boldsymbol{F}-\boldsymbol{I}, 500 \mathrm{~nm}$.

mice at P42 (Fig. 2A-E). The qualitative (Fig. 2A-D) and quantitative observations (Fig. $2 E$ ) indicate there are not any differences in either the form or the height of the vestibular hair bundles in the heterozygous Ptprq $^{+/-}$mice relative to those seen and measured in the Ptprq ${ }^{+/+}$mice. Further morphological studies were therefore restricted to comparing hair bundle features and numbers in Ptprq ${ }^{+-}$and Ptprq ${ }^{-1-}$ mice.

Hair bundle morphology in the striolar and extrastriolar regions of the utricle in Ptprq ${ }^{+1-}$ and Ptprq ${ }^{-1-}$ mice at P2, P5, $\mathrm{P} 16, \mathrm{P} 42$, and $\mathrm{P} 177$, as observed in phalloidin-stained wholemount preparations, is illustrated for the AMES and STR regions in Figures 3 and 4, respectively. Quantitative measurements for hair bundle height (length of the tallest stereocilia in the bundle) from all six regions studied, and for the profile area and numbers of hair bundles in the AMES and STR regions of the utricle at P2, P5, P16, P42, P177, and P380 are presented in Figure 5. At P2, hair bundles of phalloidin-stained utricles of Ptprq ${ }^{-1-}$ mice do not look obviously different from those of tprq $^{+1}$ - siblings (Figs. $3 A, B, 4 A, B)$, with the exception of the stereocilia appearing thicker in the striolar hair bundles of the mutant (Fig. $4 A, B$ ). A difference in hair bundle height is not seen in any region at $\mathrm{P} 2$ (Figs. $3 A, B, 4 A, B, 5 A$ ), although the average hair bundle profile area at P2 is slightly (but not significantly) reduced in the extrastriolar regions (as shown for the AMES region in Fig. 5B), sug- gesting a reduction of stereocilia number in the hair bundles of mutants.

At P5, although there is not a significant difference in hair bundle height (Fig. 5A), the hair bundles in the utricle of the Ptprq ${ }^{-1-}$ mice have stereocilia that appear thicker and are fewer in number than those of Ptprq ${ }^{+/-}$controls (Figs. 3C,D, 4C,D). The increased thickness of the stereocilia is most noticeable in the striolar region (Fig. 4C,D), and a reduction in hair bundle profile area is observed in both the extrastriolar and striolar regions by this stage (Fig. $5 B$ ). By P16, hair bundles in the extrastriolar regions of the utricle in the Ptprq ${ }^{-1-}$ mice are significantly taller relative to those in the Ptprq ${ }^{+1-}$ mice (Figs. $3 E, F, 5 A$ ). In contrast, between $\mathrm{P} 5$ and P16, hair bundle height does not increase in the striolar region in the Ptprq ${ }^{-1-}$ mouse (Figs. 4E, F, 5A). While the mutant hair bundle phenotype becomes more obvious in all regions by $\mathrm{P} 42$ (Figs. 3G, $H, 4 G, H$ ), hair bundle height does not exhibit a significant increase between the P16 and P42 stages (Fig. 5A) in any area of either the Ptprq ${ }^{+1-}$ or Ptprq ${ }^{-1-}$ mouse and essentially remains constant in the mutant between P16 and $\mathrm{P} 380$, the latest stage examined in the current study. A reduction in hair bundle profile area in the Ptprq ${ }^{-1-}$ mutants becomes less obvious in extrastriolar regions after P16 (Fig. 5B, AMES) while becoming more pronounced in the striolar region (Fig. 5B, STR). This difference can be explained by the increased hair bundle 

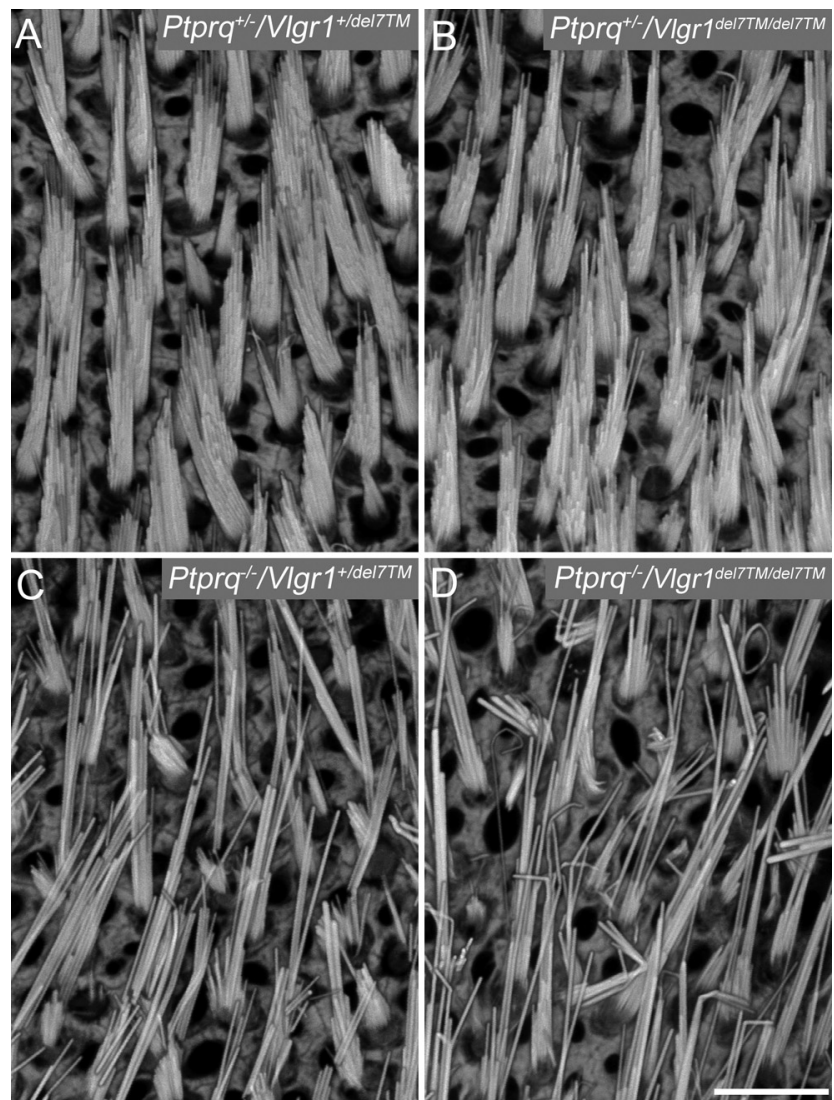

$\mathrm{E}$

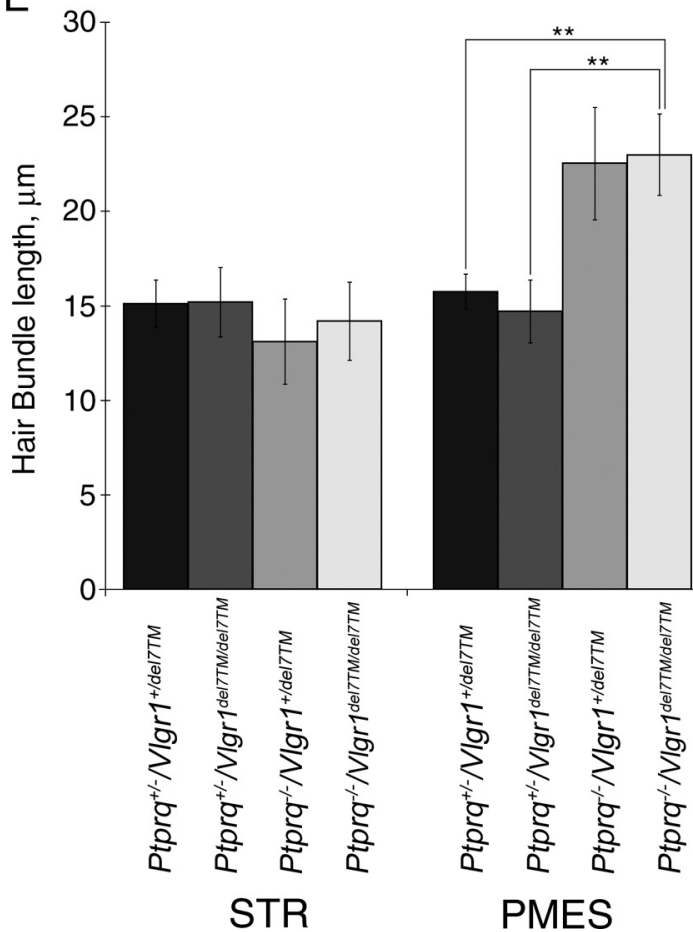

Figure 9. Hair bundle morphology and length quantification in VIgr1/Ptprq double mutants. $A-D$, Phalloidin-stained hair bundles from the PMES region of P32 mouse utricles from Ptprq ${ }^{+/-}$/

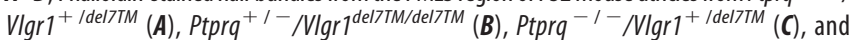
Ptprq $^{-1-}$ NIgr $^{\text {del7TM/delTTM }}(\boldsymbol{D})$ mice. Disrupted hair bundles are only seen in mice that are homozygous for the Ptprq mutation (C, D). Scale bar: (in $\boldsymbol{D}) \boldsymbol{A}-\boldsymbol{D}, 10 \mu \mathrm{m}$. $\boldsymbol{E}$, Comparison of hair bundle lengths at P32 in the STR and PMES regions of Ptprq ${ }^{+1-} / \mathrm{Nlgr}^{+} /$del/TM, $\mathrm{Ptprq}^{+1-}$ /

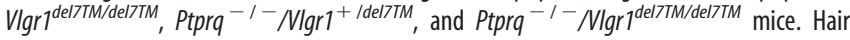
bundles are significantly longer in the PMES region of mice that are homozygous for the Ptprq height that is seen only in the extrastriolar hair bundles at these stages (Fig. 5A). Counts of hair bundle numbers indicate that, by P42 and thereafter, there may be fewer hair bundles in the extrastriolar regions in the Ptprq ${ }^{-1-}$ mutants. The differences in numbers are not, however, significant at the $p<0.05$ level (Fig. $5 C$ ).

A qualitative assessment of the other vestibular organs of the inner ear indicates all are affected to varying degrees by the loss of Ptprq. Both fusion and elongation of stereocilia are observed in the saccule of the Ptprq ${ }^{-1-}$ mouse (Fig. $6 A-D$ ). In the cristae, fusion of stereocilia is observed in the hair bundles without any obvious elongation (Fig. $6 E-H$ ). Although the very tall hair bundles seen in the peripheral regions of the cristae resemble the elongated hair bundle seen in the extrastriolar regions of the maculae of Ptprq ${ }^{-1-}$ mutants, immunolabeling of wild-type and Ptprq $^{+1-}$ cristae indicates that these hair bundles normally express Ptprq (Fig. 6I,J). As in the striolar regions of the maculae, Ptprq labeling is tightly restricted to the base of hair bundles in the central region of each crista (Fig. $6 \mathrm{~J}, \mathrm{~J}^{\prime}$ ), while it extends up the hair bundle to a greater extent in the peripheral region (Fig. $6 I, I^{\prime}$ ).

Small gaps are observed in the phalloidin staining of stereocilia in the utricular macula (Figs. 3, 7A), as have been described previously in the hair cells of noise-exposed cochleae and in the vestibular hair bundles of both wild-type and $\gamma$-actin mutant mice (Belyantseva et al., 2009). While these gaps are readily observed in the utricular maculae of the $\mathrm{Ptprq}^{-1-}$ mice (Figs. 3, 7A), they are not exclusively found in mutants and may be simply more apparent in the hair bundles of mutants as the stereocilia are fewer in number and thicker. Cytocauds, which are aberrant F-actin bundles that project down from the hair bundle into the cell soma in some mutants and were first described by Flock et al. (1979) in the hair cells of the waltzing guinea pig, are also a common feature of the hair cells in the Ptprq $^{-1-}$ mutant (Fig. 7B-D). As with the gaps seen in the phalloidin staining of stereocilia, cytocauds are not a feature that is exclusively found in the mutants.

Scanning and transmission electron microscopy both provide evidence for the fusion of stereocilia in the utricular hair bundles of the Ptprq ${ }^{-I-}$ mutant mouse (Fig. 8). Adjacent stereocilia are often seen to be in very close apposition in the hair bundles of the Ptprq $^{-1-}$ mutant mice (Fig. 8D, G), probably due to the reduced density of the cell coat in the mutant. This close apposition may be a prelude to fusion. Fusion is more commonly encountered in the taller stereocilia of the hair bundle (Fig. $8 B, D, G, I$ ). The kinocilium, a component of the hair bundle that does not express Ptprq, does not fuse with the tallest stereocilia in the Ptprq ${ }^{-/}$mutant mice (Fig. $8 G, I)$. Ultrastructural abnormalities of the stereocilium that may correspond to the gaps in phalloidin staining readily observed in Ptprq $^{-1-}$ mice are shown in Figure $8 \mathrm{E}$. In one case, the actin bundle within the core is narrowed and detached from the constricted ensheathing membrane of the stereocilium (Fig. 8E, short arrow). In the other instance, a band of dense, fragmented material is observed that appears to replace or obscure the F-actin filaments (Fig. $8 E$, long arrow).

As shaft connectors are one of three known subsets of interstereocilial lateral links, we examined whether the effect of losing shaft connectors might be exacerbated by the loss of ankle links. Vlgr1 is one

allele $(p<0.001)$ than in Ptprq ${ }^{+/-} /$Vlgr $^{+/ \text {del } 7 T M}$ mice. No significant difference in hair

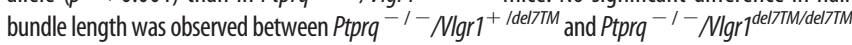
mice, indicating that mutations in Vlgr1 do not affect hair bundle length. Significant differences in hair bundle length were not observed in the STR region between any of the four genotypes. **Indicates that data sets are significantly different at the $0.1 \%$ level. $p>0.05$ was considered nonsignificant. 
component of ankle links. Its loss leads to defects in hair bundle structure in cochlear, but not in vestibular, hair bundles despite its expression in the vestibular system (McGee et al., 2006). Hair bundles in the extrastriolar (Fig. 9) and striolar (data not shown) regions of the Ptprq ${ }^{+/-} /$Vlgr $1^{+/ \text {del } 7 T M}$ (Fig. 9A) and Ptprq $^{+/-} /$Vlgr $1^{\text {del7TM/del7TM }}$ (Fig. 9B) mice are of similar morphological appearance, as expected from previous data showing the lack of a phenotype in the vesti-

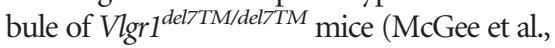
2006). Likewise, the loss of Vlgr1 in Ptprq ${ }^{-1-}$ / Vlgr1 ${ }^{\text {del7TM/del7TM }}$ mice (Fig. 9D) does not obviously lead to any further disruption in hair bundle structure relative to that seen in Ptprq ${ }^{-/-} / V_{l g r} 1^{+/ \text {del } 7 T M}$ mice (Fig. 9C). A quantitative analysis of hair bundle heights in striolar and extrastriolar regions of the single- and double-mutant mice (Fig. $9 E$ ) also reveals that hair bundle heights in the Ptprq $^{-1-} / \operatorname{Vlg} 1^{\text {del7TM/del7TM }}$ mice are not any greater than those observed in the Ptprq $^{-/-} /$Vlgr $1^{+/ \text {del } 7 T M}$ mice.

VsEPs could only be detected in 3 of 15 Ptprq ${ }^{-1-}$ mutants tested at $\sim 3$ months of age and were completely absent from the other 12 that were tested (Fig. 10A-C). VsEPs for the three mutants with measurable responses were still quite abnormal evidencing significantly prolonged latencies $(1.72 \pm 0.19 \mathrm{~ms})$ and elevated thresholds $(-0.50 \pm 1.73 \mathrm{~dB}$ re: $1.0 \mathrm{~g} / \mathrm{ms})$ compared with Ptprq ${ }^{+/-}$and Ptprq ${ }^{+/+}$mice. Indeed, on average, all 15 Ptprq $^{+/-}$mice had measurable VsEPs with peak latencies (1.38 \pm $0.05 \mathrm{~ms}$ for $\mathrm{P} 1)$ and amplitudes $(0.51 \pm 0.18$ $\mu \mathrm{V}$ for P1-N1) similar to Ptprq ${ }^{+/+}$animals $(1.39 \pm 0.06 \mathrm{~ms}$ for $\mathrm{P} 1$ and $0.58 \pm 0.14 \mu \mathrm{V}$ for P1-N1). Thresholds, however, for Pt$\mathrm{prq}^{+1-}$ mice were variable, ranging from -1.5 to $-10.5 \mathrm{~dB}$ re: $1.0 \mathrm{~g} / \mathrm{ms}$ and averaged $-6.50 \pm 2.70 \mathrm{~dB}$ compared with an average threshold of $-8.75 \pm 1.50 \mathrm{~dB}$ for wild types. The large range of thresholds for Ptprq $^{+1-}$ mice suggests some variability in gravity receptor sensitivity among heterozygotes, although on average heterozygotes were not significantly different from wild types. Overall, VsEPs for Ptprq ${ }^{-1-}$ mutants suggest severe to profound gravity receptor dysfunction.

We assessed a litter of P177 mice from the Ptprq colony composed of five heterozygotes and four homozygotes, and a group of eight mice (four heterozygotes and four mutants) at P360 (those used for the morphological analysis at P380) using a variety of simple behavioral tasks designed to test performance of the vestibular system. These tests included monitoring behavior when suspended by the tail, when placed on a rotating rod, and when swimming (Fig. 10D-G). Subtle defects that were indicative of the mutant genotype in seven of eight animals were

A
Ptprq $^{+-}$
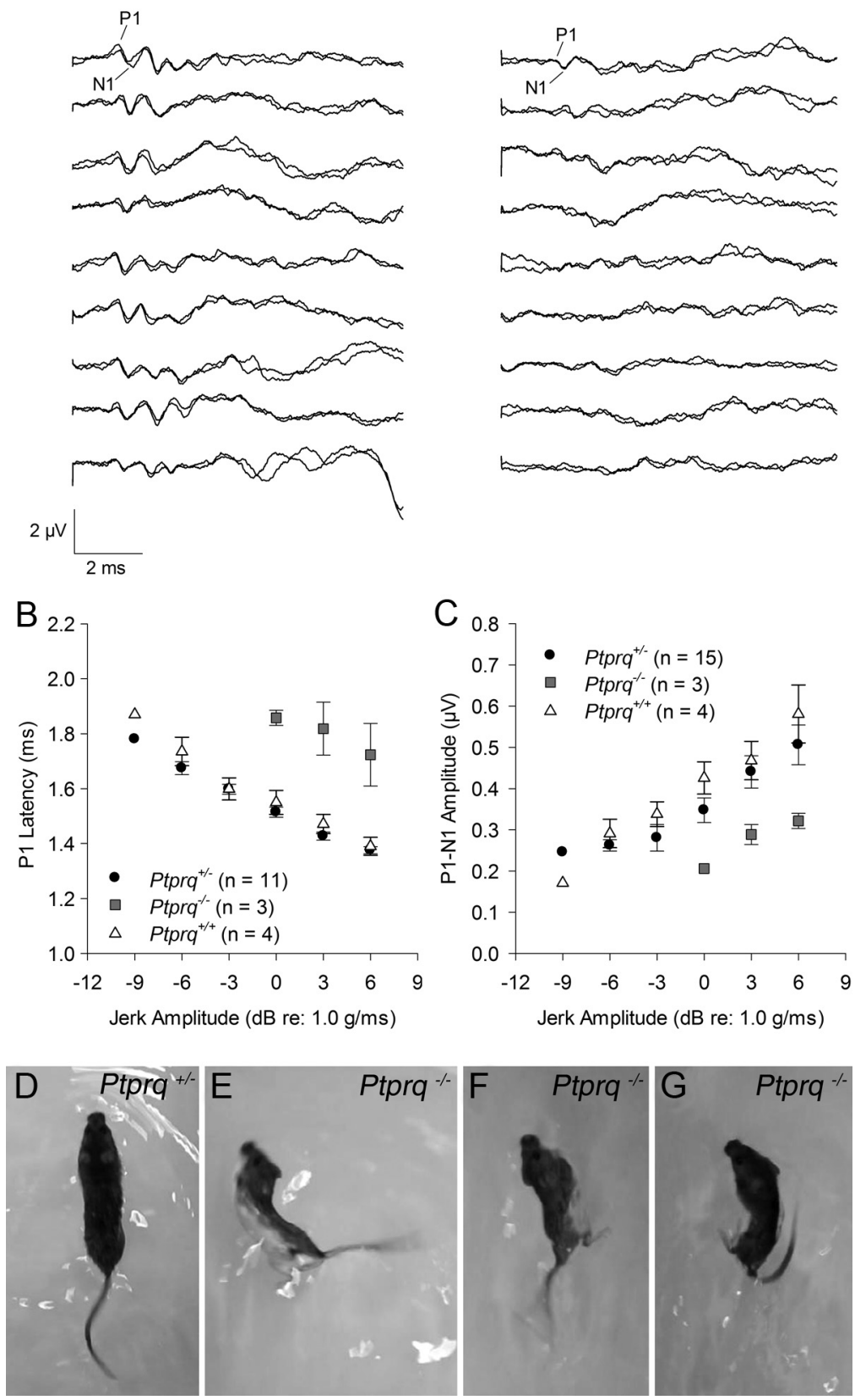

Figure 10. Vestibular evoked potentials and swimming behavior. Representative VsEP waveforms recorded at the maximum stimulus level ( $+6 \mathrm{~dB}$ re: $1.0 \mathrm{~g} / \mathrm{ms}$ ) for nine Ptprq ${ }^{+/-}$and nine Ptprq ${ }^{-/-}$mice $(\boldsymbol{A})$. Waveforms from Ptprq ${ }^{+/+}$mice are not shown but were similar to the Ptprq ${ }^{+1-}$ waveforms. Most Ptprq ${ }^{+1-}$ mice showed robust response peaks; however, a few showed reduced amplitudes with elevated thresholds. VsEPs were absent from the majority of Ptprq ${ }^{-1-}$ mice at the maximum stimulus level. Those mutants with measurable VsEPs showed significantly prolonged P1 latencies $(\boldsymbol{B})$ and reduced P1-N1 amplitudes compared with heterozygotes and wild types $(\boldsymbol{C})$. Single frames from movies of swimming behavior in a Ptprq ${ }^{+1-}$ mouse (D) and three different Ptprq ${ }^{-1-}$ mice $(\boldsymbol{E}-\boldsymbol{G})$ at 12 months of age. Heterozygotes exhibit normal swimming behavior, with an elongated, balanced posture and tail extending caudally like a corkscrew $(\boldsymbol{D})$. Abnormal swimming behavior in mutants is characterized by rolling to one side or other $(\boldsymbol{E}, \boldsymbol{F})$ and by the tail curling back toward the head $(\boldsymbol{G})$.

only observed in swimming behavior. In the swimming tests, the mutants showed a tendency to swim in circles and/or roll to one side or the other (Fig. $10 E-G$ ). Swimming behavior was normal in all nine heterozygous animals that were tested.

\section{Discussion}

The results of this study provide evidence that the absence of Ptprq leads to defects in the structure and function of vestibular 
hair cells. The morphological defects manifest to a large degree during the first 2 weeks of postnatal life, and by 3 months of age vestibular evoked potentials cannot be detected or have a considerably elevated threshold.

A number of factors contribute to the recognition of this phenotype, including an improved method for tissue preparation and a quantitative analysis of hair bundle structure in defined regions of the utricle. In an earlier study (Goodyear et al., 2003), utricular maculae were fixed after they had been dissected from the labyrinth, and after the otoconial membranes had been peeled away with fine forceps. This methodology can lead to considerable hair bundle damage in wild-type mice and obscures the mutant phenotype. By fixing the entire labyrinth overnight before dissecting out the macula, and by clearing the otoconia with EDTA rather than physically removing the otoconial membrane, it was possible to prevent most of the damage that makes it hard to detect the consequences of the mutation. Analysis of precisely defined areas also helped in distinguishing the early, subtle defects from the normal variation in hair bundle size and shape that occurs between different regions of the utricle (Rüsch et al., 1998; Li et al., 2008). Finally, mounting the tissue with the otoconial membrane intact flattens the hair bundles against the surface of the epithelium (Fig. 7C) and allows one to make accurate measurements and comparisons of hair bundle length.

In the absence of Ptprq, vestibular hair bundles initially have a smaller profile, have stereocilia that become fused, and, in the extrastriolar regions, have stereocilia that become elongated relative to those in heterozygous controls. While hair bundle elongation is a phenotype that is only seen in extrastriolar hair cells, the fusion of stereocilia in mutants is a feature common to both striolar and extrastriolar hair cells, although it is somewhat more extreme in hair cells of the striolar region. As suggested previously, Ptprq and the shaft "connectors" with which it is associated may prevent the fusion of stereocilia rather than promoting their cohesion. Although fusion is more prominent toward the tips of taller stereocilia, it may initiate around the base of the hair bundle. The loss of Ptprq may lead to a detachment of the apical membrane of the hair cell from the underlying actin cytoskeleton, thus allowing the membranes of adjacent stereocilia to "zipper" upward, as has been suggested for the fusion of stereocilia that is seen in the hair cells of the myosin VI-deficient Snell's waltzer mouse (Self et al., 1999; Karolyi et al., 2003). While it has been suggested that the minus-end-directed actin-based motor myosin VI interacts with Ptprq, and while this may explain the similarities in the phenotypes of the Ptprq ${ }^{-1-}$ and Snell's waltzer mice (Sakaguchi et al., 2008), there is no firm evidence that Ptprq and myosin VI interact, either directly or indirectly.

Elongated stereocilia are only seen in the extrastriolar region of the Ptprq ${ }^{-1-}$ utricle where the hair bundles would normally have Ptprq distributed in a fairly uniform manner over their entire length. Although the exact function of Ptprq in the hair bundle remains unknown, its potential ability to regulate PIP2 levels (Oganesian et al., 2003) and therefore the kinetics of actin polymerization may explain such a lengthening. Ptprq may prevent or reduce actin polymerization at the distal ends of the stereocilia in extrastriolar cells, and its loss may then lead to their elongation. As Ptprq is absent from the distal ends of striolar hair bundles, other proteins would have to play a similar role in these hair bundles. Elongation and fusion of stereocilia are features common to both the Snell's waltzer and the Ptprq mouse mutants, and it is plausible that these two phenomena may be correlated. In striolar hair cells, however, the fusion of stereocilia occurs without elongation, so fusion does not always drive elongation.
Ptprq is not expressed on the surface of the kinocilium, and the kinocilium does not fuse with the stereocilia in the Ptprq ${ }^{-1-}$ hair bundles. Instead, it remains attached to them via kinocilial links. Fusion therefore only involves the stereocilia, the elements that normally express Ptprq, and other proteins must prevent the kinocilium from fusing with the stereocilia. While is has been suggested that the kinocilial links may act as a parallel mechanotransduction system (Shin et al., 2005; Goodyear et al., 2010), vestibular evoked potentials could not be detected in the majority of Ptprq $^{-1-}$ mice at $\sim 3$ months of age, and a recent study has shown that balance function is normal in mice in which the CD2 isoform of Pcdh 15 is not expressed and in which the kinocilium is detached from the stereocilia (Webb et al., 2011).

The lack of an obvious overt behavioral phenotype in Ptprq ${ }^{-1-}$ mice is somewhat surprising considering the profound dysfunction revealed by measurements of VsEPs. It could be the case that some sensory input from the maculae is still reaching the brain, but not with sufficient synchrony to produce a compound action potential in far-field recordings. The electronystagmography findings in DFNB84 patients (see below) would suggest severe horizontal canal dysfunction. The extent of dysfunction in the semicircular canals of the Ptprq ${ }^{-1-}$ mice is not known and sensory input from this source may be sufficient to account for the lack of an overt behavioral phenotype. Compensation by visual and/or tactile stimuli may alternatively account for the lack of an obvious defect, and behavioral defects are observed during swimming, a condition in which the proprioceptive input is considered to be reduced. Some level of vestibular input must be present during the early stages of development, possibly before the fusion of the stereocilia, for reasonably competent swimming and other balance behaviors to have developed. If macular function were absent at birth, as is the case with the otoconia-deficient mutants Nox3 and Otop1 (Jones et al., 1999, 2004), then the Ptprq $^{-1-}$ mutants would be unable to swim despite compensation from other sensory systems.

Delayed motor development and a complete absence of an electronystamographic response in caloric or rotatory tests in later life have been reported for DFNB84 patients homozygous for a nonsense mutation generating an early premature stop codon ( $p$. Tyr497X) in PTPRQ. Although patients homozygous for a missense mutation (p.Arg457Gly) in PTPRQ had normal motor development, severe hyporeflexia was detected by electronystamography at $7-13$ years of age (Schraders et al., 2010). The vestibular hair bundle defects and the severe reduction in VsEPs we describe for the Ptprq ${ }^{-1-}$ mouse mutants within 1-3 months of age are therefore consistent with vestibular dysfunction reported for DFNB84 patients that are homozygous for the nonsense mutation in PTPRQ. Our data would suggest that DFNB84 may include macular dysfunction in addition to the semicircular canal deficits observed with electronystagmography. To our knowledge, clinical measures of macular function, such as vestibular evoked myogenic potentials, have not been reported for DFNB84 patients.

\section{References}

Ahmed ZM, Riazuddin S, Bernstein SL, Ahmed Z, Khan S, Griffith AJ, Morell RJ, Friedman TB, Riazuddin S, Wilcox ER (2001) Mutations of the protocadherin gene PCDH15 cause Usher syndrome type 1F. Am J Hum Genet 69:25-34.

Ahmed ZM, Goodyear R, Riazuddin S, Lagziel A, Legan PK, Behra M, Burgess SM, Lilley KS, Wilcox ER, Riazuddin S, Griffith AJ, Frolenkov GI, Belyantseva IA, Richardson GP, Friedman TB (2006) The tip-link antigen, a protein associated with the transduction complex of sensory hair cells, is protocadherin-15. J Neurosci 26:7022-7034.

Alagramam KN, Murcia CL, Kwon HY, Pawlowski KS, Wright CG, Woychik RP (2001) The mouse Ames waltzer hearing-loss mutant is caused by mutation of Pcdh15, a novel protocadherin gene. Nat Genet 27:99-102. 
Belyantseva IA, Perrin BJ, Sonnemann KJ, Zhu M, Stepanyan R, McGee J, Frolenkov GI, Walsh EJ, Friderici KH, Friedman TB, Ervasti JM (2009) $\gamma$-Actin is required for cytoskeletal maintenance but not development. Proc Natl Acad Sci U S A 106:9703-9708.

Bork JM, Peters LM, Riazuddin S, Bernstein SL, Ahmed ZM, Ness SL, Polomeno R, Ramesh A, Schloss M, Srisailpathy CR, Wayne S, Bellman S, Desmukh D, Ahmed Z, Khan SN, Kaloustian VM, Li XC, Lalwani A, Riazuddin S, Bitner-Glindzicz M, et al. (2001) Usher syndrome 1D and nonsyndromic autosomal recessive deafness DFNB12 are caused by allelic mutations of the novel cadherin-like gene CDH23. Am J Hum Genet 68:26-37.

Flock A, Cheung H, Wersäll J (1979) Pathological actin in vestibular hair cells of the waltzing guinea pig. Adv Otorhinolaryngol 25:12-16.

Goodyear R, Richardson G (1992) Distribution of the $275 \mathrm{kD}$ hair cell antigen and cell surface specialisations on auditory and vestibular hair bundles in the chicken inner ear. J Comp Neurol 325:243-256.

Goodyear RJ, Legan PK, Wright MB, Marcotti W, Oganesian A, Coats SA, Booth CJ, Kros CJ, Seifert RA, Bowen-Pope DF, Richardson GP (2003) A receptor-like inositol lipid phosphatase is required for the maturation of developing cochlear hair bundles. J Neurosci 23:9208-9219.

Goodyear RJ, Forge A, Legan PK, Richardson GP (2010) Asymmetric distribution of cadherin 23 and protocadherin 15 in the kinocilial links of avian sensory hair cells. J Comp Neurol 518:4288-4297.

Jones SM, Erway LC, Bergstrom RA, Schimenti JC, Jones TA (1999) Vestibular responses to linear acceleration are absent in otoconia-deficient C57BL/6JEi-het mice. Hear Res 135:56-60.

Jones SM, Erway LC, Johnson KR, Yu H, Jones TA (2004) Gravity receptor function in mice with graded otoconial deficiencies. Hear Res 191:34-40.

Karolyi IJ, Probst FJ, Beyer L, Odeh H, Dootz G, Cha KB, Martin DM, Avraham KB, Kohrman D, Dolan DF, Raphael Y, Camper SA (2003) Myo15 function is distinct from Myo6, Myo7a and pirouette genes in development of cochlear stereocilia. Hum Mol Genet 12:2797-2805.

Kazmierczak P, Sakaguchi H, Tokita J, Wilson-Kubalek EM, Milligan RA, Müller U, Kachar B (2007) Cadherin 23 and protocadherin 15 interact to form tip-link filaments in sensory hair cells. Nature 449:87-91.

Li A, Xue J, Peterson EH (2008) Architecture of the mouse utricle: macular organization and hair bundle heights. J Neurophysiol 99:718-733.

McGee J, Goodyear RJ, McMillan DR, Stauffer EA, Holt JR, Locke KG, Birch DG, Legan PK, White PC, Walsh EJ, Richardson GP (2006) The very large G-protein-coupled receptor VLGR1: a component of the ankle link complex required for the normal development of auditory hair bundles. J Neurosci 26:6543-6553.

McMillan DR, White PC (2004) Loss of the transmembrane and cytoplasmic domains of the very large G-protein-coupled receptor-1 (VLGR1 or Mass1) causes audiogenic seizures in mice. Mol Cell Neurosci 26:322-329.

Michalski N, Michel V, Bahloul A, Lefèvre G, Barral J, Yagi H, Chardenoux S, Weil D, Martin P, Hardelin JP, Sato M, Petit C (2007) Molecular characterization of the ankle-link complex in cochlear hair cells and its role in the hair bundle functioning. J Neurosci 27:6478-6488.

Mock B, Jones TA, Jones SM (2011) Gravity receptor aging in the CBA/CaJ strain: a comparison to auditory aging. J Assoc Res Otolaryngol 12:173-183.

Nayak GD, Ratnayaka HS, Goodyear RJ, Richardson GP (2007) Development of the hair bundle and mechanotransduction. Int J Dev Biol 51:597-608.

Nayak G, Goodyear RJ, Legan PK, Noda M, Richardson GP (2011) Evidence for multiple, developmentally regulated isoforms of Ptprq on hair cells of the inner ear. Dev Neurobiol 71:129-141.

Oganesian A, Poot M, Daum G, Coats SA, Wright MB, Seifert RA, BowenPope DF (2003) Protein tyrosine phosphatase RQ is a phosphatidylinositol phosphatase that can regulate cell survival and proliferation. Proc Natl Acad Sci U S A 100:7563-7568.

Reynolds ES (1963) The use of lead citrate at high $\mathrm{pH}$ as an electron-opaque stain in electron microscopy. J Cell Biol 17:208-212.

Rüsch A, Lysakowski A, Eatock RA (1998) Postnatal development of type I and type II hair cells in the mouse utricle: acquisition of voltage-gated conductances and differentiated morphology. J Neurosci 18:7487-7501.

Sakaguchi H, Tokita J, Naoz M, Bowen-Pope D, Gov NS, Kachar B (2008) Dynamic compartmentalization of protein tyrosine phosphatase receptor $\mathrm{Q}$ at the proximal end of stereocilia: implication of myosin VI-based transport. Cell Motil Cytoskeleton 65:528-538.

Schraders M, Oostrik J, Huygen PL, Strom TM, van Wijk E, Kunst HP, Hoefsloot LH, Cremers CW, Admiraal RJ, Kremer H (2010) Mutations in PTPRQ are a cause of autosomal-recessive nonsyndromic hearing impairment DFNB84 and associated with vestibular dysfunction. Am J Hum Genet 86:604-610.

Schwander M, Kachar B, Müller U (2010) Review series: the cell biology of hearing. J Cell Biol 190:9-20.

Self T, Sobe T, Copeland NG, Jenkins NA, Avraham KB, Steel KP (1999) Role of myosin VI in the differentiation of cochlear hair cells. Dev Biol 214:331-341.

Shahin H, Rahil M, Abu Rayan A, Avraham KB, King MC, Kanaan M, Walsh $T$ (2010) Nonsense mutation of the stereociliar membrane protein gene PTPRQ in human hearing loss DFNB84. J Med Genet 47:643-645.

Shin JB, Adams D, Paukert M, Siba M, Sidi S, Levin M, Gillespie PG, Gründer S (2005) Xenopus TRPN1 (NOMPC) localizes to microtubule-based cilia in epithelial cells, including inner-ear hair cells. Proc Natl Acad Sci U S A 102:12572-12577.

Siemens J, Lillo C, Dumont RA, Reynolds A, Williams DS, Gillespie PG, Müller U (2004) Cadherin 23 is a component of the tip link in hair-cell stereocilia. Nature 428:950-955.

Söllner C, Rauch GJ, Siemens J, Geisler R, Schuster SC, Müller U, Nicolson T, Nicolson T (2004) Mutations in cadherin 23 affect tip links in zebrafish sensory hair cells. Nature 428:955-959.

Verpy E, Masmoudi S, Zwaenepoel I, Leibovici M, Hutchin TP, Del Castillo I, Nouaille S, Blanchard S, Lainé S, Popot JL, Moreno F, Mueller RF, Petit C (2001) Mutations in a new gene encoding a protein of the hair bundle cause non-syndromic deafness at the DFNB16 locus. Nat Genet 29:345-349.

Verpy E, Weil D, Leibovici M, Goodyear RJ, Hamard G, Houdon C, Lefèvre GM, Hardelin JP, Richardson GP, Avan P, Petit C (2008) Stereocilindeficient mice reveal the origin of cochlear waveform distortions. Nature 456:255-258.

Verpy E, Leibovici M, Michalski N, Goodyear RJ, Houdon C, Weil D, Richardson GP, Petit C (2011) Stereocilin connects outer hair cell stereocilia to one another and to the tectorial membrane. J Comp Neurol 519:194-210.

Webb SW, Grillet N, Andrade LR, Xiong W, Swarthout L, Della Santina CC, Kachar B, Müller U (2011) Regulation of PCDH15 function in mechanosensory hair cells by alternative splicing of the cytoplasmic domain. Development 138:1607-1617.

Weston MD, Luijendijk MW, Humphrey KD, Möller C, Kimberling WJ (2004) Mutations in the VLGR1 gene implicate G-protein signaling in the pathogenesis of Usher syndrome type II. Am J Hum Genet 74:357366. 